Original Article

\title{
Increased cortical excitability after selective REM sleep deprivation in healthy humans: A transcranial magnetic stimulation study
}

\author{
Fabio Placidi ${ }^{\text {a,* }}$, Silvana Zannino ${ }^{\mathrm{b}}$, Maria Albanese ${ }^{\mathrm{a}}$, Andrea Romigi ${ }^{\mathrm{a}, \mathrm{c}}$, Francesca Izzi ${ }^{\mathrm{a}}$, \\ Maria G. Marciani ${ }^{\text {a,b }}$, Maria G. Palmieri ${ }^{\text {a }}$ \\ a Department of Neurosciences, University of Rome Tor Vergata, Roma, Italy \\ ${ }^{\mathrm{b}}$ IRCCS Fondazione Santa Lucia, Roma, Italy \\ 'Sleep Medicine Center IRCCS “NEUROMED” Pozzilli (Is), Italy
}

\section{A R T I C L E I N F O}

\section{Article history:}

Received 27 August 2012

Received in revised form 4 November 2012

Accepted 29 November 2012

Available online $\mathrm{xxxx}$

\section{Keywords:}

REM sleep deprivation

Cortical excitability

Transcranial magnetic stimulation

Intracortical inhibition (ICI)

Cortical silent period

\begin{abstract}
A B S T R A C T
Background: REM sleep has antiepileptogenic properties whereas, its loss is known to have a proconvulsive role. However, the mechanisms underlying the proepileptogenic effects of REM sleep deprivation are yet not fully understood. The aim of our study was to evaluate the effects of selective REM sleep deprivation (SRD) on cortical excitability in healthy subjects by means of transcranial magnetic stimulation (TMS).

Methods: Ten normal subjects underwent three TMS sessions: (1) in baseline condition (BL), (2) after SRD by awakening them at each REM sleep onset and (3) after non-rapid eye movement sleep awakenings (NREM-A) as control for potential non-specific effects of interruptions. The TMS investigation included two protocols: (a) the evaluation of motor evoked potentials (MEPs) and silent period (SP) parameters, recorded in response to single pulse magnetic stimulation; (b) the evaluation of the time course of intracortical motor activity tested with paired-pulse TMS applied at inter-stimulus intervals of $1-10 \mathrm{~ms}$.

Results: After SRD the principal finding observed using single pulse TMS was a significant reduction in the duration of SP whereas, a reduction of intracortical inhibition was found, using the paired-pulse TMS. TMS parameters did not show significant changes after NREM-A with respect to BL.

Conclusions: SRD may influence cortical excitability with a reduction of inhibitory intracortical mechanisms, thus supporting the proconvulsant role of REM loss.
\end{abstract}

(c) 2012 Elsevier B.V. All rights reserved.

\section{Introduction}

Sleep and epilepsy are intimately related by mutual and complex interactions [1]. Non-REM sleep seems to have a permissive effect on seizures and EEG epileptic discharges, whereas REM sleep has an inhibitory action on both interictal and ictal paroxysms [2]. In addition, sleep deprivation is capable to induce a sensitization to seizures [3] and it is therefore, employed in clinical settings as an activation test of EEG in the diagnosis of epilepsy [4]. REM sleep has antiepileptogenic properties [5-7] whereas its loss is known to have a proconvulsive role [8-10]. However, the mechanisms underlying the proepileptogenic effects of REM sleep deprivation are yet not fully understood.

Transcranial magnetic stimulation (TMS) is a safe and non-invasive diagnostic technique for exploring the motor pathways and human motor excitability in vivo $[11,12]$. Several TMS measures

\footnotetext{
* Corresponding author. Address: Neuroscience Department, Policlinico Tor Vergata, Viale Oxford 81, 00133 Rome, Italy. Tel.: +39 0620902107; fax: +39 0620902116

E-mail address: fbplacidi@gmail.com (F. Placidi).
}

contribute to assessment, of which the excitability threshold, the amplitude of the motor evoked potential (MEP) and the silent period (SP), may be evaluated by the single pulse technique. These parameters are very sensitive for detecting both physiological and pathological conditions involving central motor pathways. In addition, more complex TMS protocols (such as paired TMS) allow the measurement of the time course of intracortical motor activity reflecting the excitability of interneuron populations intrinsic to motor cortical area [13-16].

Recently, some studies documented an impairment of cortical excitability induced by total sleep deprivation in both healthy and epileptic subjects by using TMS [17-21]. To date, the effects of Selective REM sleep Deprivation (SRD) on cortical excitability have not been explored in humans.

We aimed to study the effects of SRD on the cortical excitability in healthy subjects assessed by means of single and paired TMS.

\section{Methods}

Ten healthy right-handed volunteers (six females, four males, mean age $25.4 \pm 3.1$, range $20-30$ years), recruited from the student 
population, were included in our study and submitted to TMS. All participants were required to maintain a regular sleep schedule the week before the study sessions, as verified by actigraphy and sleep diary. During three days preceding the study as well as during the study, the subjects were instructed to abstain from alcohol and caffeinated beverages. The local ethical committee for research on human subjects approved the study protocol, and written informed consent was obtained from the subjects before the study.

TMS, using single or paired stimuli, was delivered via a focal butterfly-shaped coil connected to one or two Magstim 200 stimulator units through a Bi-stim module (Magstim, Whitland, Dyfed, UK). We always stimulated the non-dominant hemisphere, because it has been proposed that there is an asymmetry in the excitability of cortical inhibitory mechanism between the two hemispheres [22]. Subjects were classified as right or left handed according to their description of the hand used for writing.

Motor evoked potentials were recorded from the opponens pollicis of the left hand via surface electrodes applied in a belly-tendon montage.

All subjects underwent three TMS sessions, so that motor cortex excitability was separately assessed in three different conditions: (1) after a full night of spontaneous sleep (baseline, BL), (2) after a night of selective SRD and (3) after a night with non-rapid eye movement sleep awakenings (NREM-A) as control for potential nonspecific effects of sleep interruptions.

Each TMS session was preceded by two nights of lab polysomnography (PSG). The first night was considered as adaptation. During the SRD nights, performed according to Endo's criteria [23], the subjects were awakened at the first sign of REM sleep and required to sit up in bed for two minutes. The criteria for interventions were 20 seconds epoch of desynchronized EEG without spindles or Kcomplexes and the concomitant reduction of the tonic EMG amplitude regardless of the occurrence of rapid eye movements. In detail, subjects were awakened by having their names called over an intercom. Simultaneously, the experimenter entered the recording chamber with the lights off and gently shook the subject until receiving a response. Volunteers were kept awake for two minutes by asking them incidental questions (e.g., name the days of the week), to avoid the immediate relapse into REM sleep.

The NREM-A nights served as a control for the repeated awakenings. During NREM-A nights, the subjects were awakened from one to two NREM sleep stages and kept awake for two minutes using the same experimental protocol as for SRD [25]. In the NREM-A session care was taken to minimize interference with slow wave sleep.

During all sleep nights, the EEG (F4, C4, O2, F3, C3, O1, A2, and A1), submental EMG and EOG were recorded. Sleep stages were visually scored for 30 seconds epochs according to the criteria of Rechschaffen and Kales [24]. Sleep stage percentages were calculated over total sleep time (TST = time from sleep onset to morning awakening after the time awake during the night is subtracted). Sleep efficiency (SE) was calculated from the ratio between TST and time in bed (TIB = recording time between lights off to morning awakening).

The interval between the sessions was not strictly predefined, but it was not shorter than one week. In order to prevent the effect of circadian factors, the TMS sessions were performed always in the late morning (11-12 a.m.). To avoid the hormonal effects on cortical excitability, all females had testing sessions performed during the same phase of the menstrual cycle [25]. To control vigilance fluctuations during the TMS sessions subjects were co-recorded with PSG and required to stay alert with eyes open and body muscles relaxed. Subject behavior was continuously checked by the technician throughout the recording.

The order of the sessions (BL versus SRD) was counterbalanced across subjects, although the SRD preceded the NREM-A sessions. This allowed a number of awakenings similar for SRD and NREMA nights.
Each TMS recording session included two protocols: (1) Singlepulse TMS, with evaluation of MEPs, Motor threshold and of SP parameters [11,12] and (2), Paired-pulse stimulation, with evaluation of time course of intracortical motor activity tested at 1-10 ms inter-stimulus intervals [13].

\subsection{Single-pulse TMS protocol}

The optimal scalp position of the coil was assessed by moving the coil in one centimeter steps over the presumed hand motor area. Coil location was determined as the site that elicited the optimal MEP amplitude during muscle relaxation with the lowest threshold. The coil was held tangential to the skull with the handle pointing backwards at $45^{\circ}$ lateral to the midline. Usually the 'optimal' responses were elicited when the coil was placed five to six centimeters along the coronal line from $\mathrm{Cz}$ point (10-20 International System) $[11,26]$.

For motor threshold measurement, MEPs were recorded during relaxation of the target muscle $[11,12]$. At threshold TMS value, a moderate contraction allowed the detection of both MEP and silent period parameters in the $500 \mathrm{~ms}$ following TMS. Audio-visual electromyographic feedback was provided to control for muscle relaxation and contraction.

The mean of three trials was used to define the following parameters and each trial was the average of three consecutive motor responses [27]:

- Motor threshold (\%), expressed as the percentage of the stimulator's maximal output, defined as the intensity required to elicit detectable MEPs with amplitudes of $0.05-0.15 \mathrm{mV}$ in $50 \%$ of the stimuli [11].

- MEP amplitude (mV), defined as the peak-to-peak amplitude between the largest negative and positive deflections following stimulus onset [11].

- Silent period duration (ms), measured from the MEP to the rebound of voluntary EMG activity (absolute duration of silent period) [27-28].

\subsection{Paired-pulse TMS protocol}

MEPs were recorded during complete relaxation of the target muscle. A conditioning test design was used to investigate the time course of intracortical motor activity. Paired stimuli were applied with conditioning pulses delivered between 1-10 ms before test stimulation. The intensity of the conditioning pulse was maintained below the threshold necessary for evoking responses in contracted muscles (70\% of the individual resting motor threshold). Test pulses were delivered suprathreshold for eliciting relaxed MEPs (110-120\% of the individual resting motor threshold). In each block test and conditioning pulses at the different inter-stimulus intervals were randomly mixed. In order to achieve a complete set of inter-stimulus intervals, several blocks of trials were performed. Each block included sixteen trials, with eight having the test stimulus alone (unconditioned MEP) and eight having pairs of condition-test pulses delivered at one of the 10 inter-stimulus intervals (conditioned MEP). The sequence began and ended with the unconditioned trials, with the conditioned MEP trials in between $[13,29]$. Mean amplitudes of unconditioned and conditioned MEPs were calculated separately for each inter-stimulus interval. The amplitude of conditioned MEPs was expressed as the percentage of unconditioned MEPs amplitude. The time-course was defined as the mean amplitude variation of conditioned MEPs (expressed as the percentage of "unconditioned" MEPs amplitude) at each inter-stimulus interval. 


\subsection{Data analysis}

For statistical analysis the SPSS program (release 16.0.2, Chicago, USA) for Windows was used. Motor threshold, MEP amplitude, and SP duration differences between conditions (BL versus SRD versus NREM-A) were tested using paired $t$-tests, with a Bonferroni correction of $p$ values when required.

For PSG data a two-way analysis of variance (ANOVA) with repeated measures was used with condition (basal versus SRD versus NREM-A) and sleep parameters as within factors. The same analysis was separately utilized for paired pulse data with condition (basal versus SRD versus NREM-A) and inter-stimulus intervals $(1-10 \mathrm{~ms})$ as within factors. Provided the $F$ was significant $(p<0.05)$, a post-hoc analysis was performed by using the Tukey HDS test. Differences at $p<0.05$ were considered significant.

\section{Results}

\subsection{PSG data}

PSG data are expressed in Table 1. As expected, REM sleep was significantly reduced during SRD with respect to BL and NREM-A. TST was significantly reduced during both SRD and NREM-A conditions with respect to $\mathrm{BL}$, but this parameter was not statistically different between SRD and NREM-A nights. Other nocturnal sleep data were not significantly different between conditions.

\subsection{Single-pulse TMS: MEPs, motor threshold, and silent period}

Comparison between excitability threshold in BL, SRD and NREMA conditions showed no significant difference. Similarly, amplitude of 'contracted' MEPs was not significantly different between conditions.

By contrast, SP duration in the SRD condition was significantly shorter than in the baseline condition $(55.8 \mathrm{~ms} \pm 15.8$ versus $68.9 \mathrm{~ms} \pm$ 18.5 , respectively; $p=0.003$ ) and NREM-A condition ( $55.8 \mathrm{~ms} \pm 15.8$ versus $70.2 \pm 20.8$, respectively, $p=0.002$ ) (Table 2 ).

\subsection{Paired-pulse TMS data}

All the subjects showed a normal intracortical motor profile to paired TMS in the basal condition. There was a significant effect of condition $(F(2.18)=25.02 ; p<0.000006)$. In fact, the mean amplitude of conditioned MEPs after SRD was significantly higher than the mean amplitude of conditioned MEPs in basal condition (112.9\% versus $91.2 \%, p=0.0001)$ and in NREM-A condition (112.9\% versus $96.7, p=0.0003$ ).

Finally, we observed a significant ISI $x$ condition effect $(F(18.162)=$ $2.15, p=0.006$ ). Post-hoc analysis comparing inter-stimulus intervals $1-10 \mathrm{~ms}$ showed significantly higher values at ISI of two ms.

Table 1

Polysomnographic parameters in baseline, SRD and NREM-A conditions.

\begin{tabular}{lccc}
\hline & BL & SRD & NREM-A \\
\hline TST & $428 \pm 48.5^{* *}$ & $379.2 \pm 18.3$ & $388 \pm 21.9$ \\
SEI & $92.5 \pm 5.3$ & $85.8 \pm 4.5$ & $86.2 \pm 5.4$ \\
S1 & $8.7 \pm 5.02$ & $14.6 \pm 4.6$ & $8.5 \pm 2.9$ \\
S2 & $51.7 \pm 3.6$ & $59.6 \pm 5.9$ & $52.3 \pm 4.4$ \\
SWS & $20.3 \pm 4.7$ & $22.1 \pm 3.4$ & $19.2 \pm 3$ \\
REM & $19.3 \pm 4.4$ & $3.7 \pm 0.9^{*}$ & $19.9 \pm 3.8$ \\
\hline
\end{tabular}

$\mathrm{BL}=$ baseline, $\mathrm{SRD}=$ selective $\mathrm{REM}$ deprivation, NREM-A = control nights with NREM sleep awakenings, TST = total sleep time in minutes, SEI = sleep efficiency index (total sleep time/time in bed $\times 100$ ), SWS = slow wave sleep, S1, S2, SWS and REM are expressed as percentages of total sleep time.

$p<0.0001$ (Two-way ANOVA, Post-hoc Tukey HSD Test) SRD vs BL and NREM-A $p<0.05$ (Two-way ANOVA, Post-hoc Tukey HSD Test) BL vs SRD and NREM-A.
Table 2

Single-pulse TMS data in baseline, SRD and NREM-A conditions.

\begin{tabular}{lccc}
\hline & BL & SRD & NREM-A \\
\hline Motor threshold (\%) & $52.1 \pm 6.6$ & $49.7 \pm 4.1$ & $50.2 \pm 5.2$ \\
MEP amplitude (mV) & $4.6 \pm 1.2$ & $5.1 \pm 1.3$ & $4.8 \pm 1.4$ \\
Cortical silent period (ms) & $68.9 \pm 18.5$ & $55.8 \pm 15.8^{*, * * *}$ & $70.2 \pm 20.8$ \\
\hline
\end{tabular}

$\mathrm{BL}=$ baseline condition, $\mathrm{SRD}=$ selective $\mathrm{REM}$ deprivation, $\mathrm{NREM}-\mathrm{I}=$ control nights with NREM awakenings.

* Comparison between SRD vs BL, $p=0.003$.

Comparison between SRD vs NREM-A, $p=0.002$.

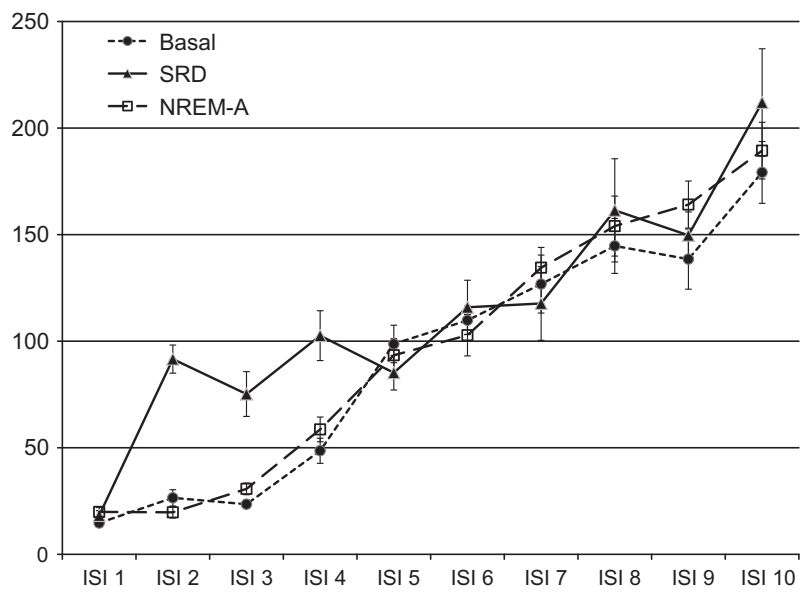

Fig. 1. Time course of intracortical motor activity tested with paired-pulse TMS. The size of the conditioned MEP, expressed as the percentage of the size of the unconditioned MEP alone is displayed at different inter-stimulus intervals (1$10 \mathrm{~ms}$ ) in baseline condition (BL, dotted line), after selective REM deprivation (SRD, solid line) and after control NREM sleep fragmentation (NREM-A, broken line). $*=$ The size of conditioned MEP at ISI of $2 \mathrm{~ms}$ after SRD was significantly higher than in baseline and NREM-A, $p<0.05$ (Post-hoc Tukey HSD Test).

after SRD with respect to baseline $(91.6 \pm 20.9$ versus $26.5 \pm 12.1$, $p=0.02)$ and to NREM-A conditions $(91.6 \pm 20.9$ versus $19.7 \pm 8.5$, $p=0.003$ ) (Fig. 1).

\section{Discussion}

The main findings of our study were the significant reduction of SP duration and the marked amplitude increase of conditioned MEPs, particularly evident at short intervals (two ms) after SRD. The latter effect is the clear expression of a reduction of the intracortical inhibition (ICI), thought to reflect the activity of inhibitory interneurons intrinsic to cortical area $4[13,14]$. Importantly, the observed effects are likely to be specifically due to SRD, given that the procedure successfully reduced REM sleep to only $3.7 \%$ over total sleep time in the SRD nights without altering SWS during the experimental nights. Furthermore, the marked reduction of both ICI and SP after SRD cannot be attributed to non-specific effects induced by sleep fragmentation, since these effects were not observed after the NREM-A nights.

The reduction of both SP duration and ICI, considered suitable markers of inhibition/facilitation balance at the cortical level [13,30] suggests an influence of SRD on neurotransmitter system of the intracortical circuitry and brainstem.

In fact, ICI is mediated by intracortical inhibitory processes in healthy subjects. Weak magnetic conditioning stimuli specifically engage cortical motor inhibitory circuits $[13,31]$. Pharmacologic studies demonstrate that ICI can be increased by drugs that enhance GABA-A transmission [15,16]. The important decrease of 
motor inhibition after SRD shown in our study may depend on modification in cortical excitability mediated by neurotransmitters, possibly the GABA-ergic system.

Otherwise, the decrease of SP duration observed in our study suggests a reduction of central motor inhibition induced by SRD. However, the silent period has a complex and partially obscure pathophysiology and reflects inhibitory activity of mainly cortical, but even subcortical and spinal circuits possibly modulated by GABAergic mechanisms [26,28,32-35]. The same multiple areas are involved in the NREM/REM sleep alternation. Pontine GABAergic mechanisms play a crucial role in the control of the motor inhibitory system operating during active sleep [36]. GABAergic REM-on and REM-off neurons localized in the ventrolateral periacqueductal gray and in the dorsal paragigantocellular reticular nucleus seem to be reciprocally involved in the regulation of paradoxical sleep [37]. Blocking GABA receptors in peduncolopontine tegmentum decreases, whereas facilitation of GABA transmission increases REM sleep in both cats [38] and rats [39]. Thus, the SRD could be able to influence one of these inhibitor mechanisms as suggested by the reduction of the SP duration.

Even though our TMS study seems to indicate a prevalent involvement of GABA-ergic processes induced by REM loss, it has to be considered that modifications of neural excitability mirror the net result of complex changes interesting different neurotransmitters, receptors and ion channels, as suggested by experimental animal studies showing that REM sleep loss provokes relevant impairment of different neurotransmitter receptor expression and functions $[40,41]$.

Sleep deprivation is a powerful activator of seizures and it is known to facilitate both generalized and focal paroxysmal EEG abnormalities $[3,4,42]$. Furthermore, an inverse correlation between REM sleep and epileptogenicity has been shown by several studies linking sleep and epilepsy.

In fact, REM sleep presents inhibitory effects on paroxysmal events in both animals and humans [5-7,43,44], whereas a proconvulsant role of REM sleep deprivation has been documented in animals [8-10]. Notably, recent animal experimental studies showed that REM sleep loss increases brain excitability through a complex cascade of intracellular and molecular events leading to increased noradrenalin levels and activation of Na-K ATPase activity [41]. On this basis, Mallick and Singh [41] have hypothesized that a fundamental function of REM sleep is the maintenance of brain excitability.

In the last decade, the effects of total sleep deprivation on human motor cortex have been explored by means of TMS in normal individuals with conflicting results, mainly due to partial different methodological protocols utilized [17-19,45]. However, an increase in cortical excitability induced by total sleep deprivation has been documented by some studies in healthy subjects $[17,18,46]$ as well as in epileptic patients $[20,21,47]$. In particular, Scalise et al. [18] reported a reduction of both SP duration and ICI after total sleep deprivation. Very recently, a combined EEG/TMS study has demonstrated a progressive increased excitability of the human frontal cortex with time awake [48].

Although an increase of neuronal brain excitability induced by REM sleep loss has been showed in animal studies [8,9,41], this effect has not been documented in humans yet.

In this study, we found a reduction of both ICI and cortical silent period suggesting an increase of brain excitability induced by SRD in healthy humans. The increased excitability induced by SRD represents a further proven of the proconvulsant role explicated by REM sleep loss. Since our results are very similar to those by Scalise et al. [18] and Kreuzer et al. [46] observed in totally sleep deprived healthy humans, we suggest that the cortical hyperexcitability induced by total sleep deprivation may be mainly due to the net contribution provoked by REM sleep loss.

\section{Conflict of interest}

The ICMJE Uniform Disclosure Form for Potential Conflicts of Interest associated with this article can be viewed by clicking on the following link: http://dx.doi.org/10.1016/j.sleep.2012.11.020.

\section{Acknowledgment}

We thank for their precious help and assistance Dr Fabrizio Cum, Dr Battista Di Gioia, Dr Cristiano Mancini, Dr Corrado Oliva, Dr Raffaella Ludovisi, Dr Nicoletta Campagna, Dr Maria Pia Giambrone, Dr Enza Tarquini and Mrs. Loredana Risita.

\section{References}

[1] Dinner DS. Effect of sleep on epilepsy. J Clin Neurophysiol 2002;19(6):504-13.

[2] Broughton RJ. Sleep and sleep deprivation studies in epilepsy. Clinical Neurophysiology of Epilepsy. Wada JR, Ellison RJ, editors. EEG Handbook, Vol. 4. Amsterdam: Elsevier; 1990. p. 89-119.

[3] Rowan AJ, Veldhuisen RJ, Nagelkerke NJD. Comparative evaluation of sleep deprivation and sedate sleep EEGs as diagnostic aids in epilepsy. Electroenceph Clin Neurophysiol 1982;101:357-64.

[4] Malow BA. Sleep deprivation and epilepsy. Epilepsy Curr 2004;4(5):193-5.

[5] Sammaritano M, Gigli GL, Gotman J. Interictal spiking during wakefulness and sleep and the localization of foci in temporal lobe epilepsy. Neurology 1991:41(2 Pt 1):290-7.

[6] Montplaisir J, Laverdiere M, Saint-Hilarie J, Rouleau I. Nocturnal sleep recording in partial epilepsy: a study with depth electrodes. J Clin Neurophysiol 1987;4(4): 383-8

[7] Kumar P, Raju TR. Seizure susceptibility decreases with enhancement of rapid eye movement sleep. Brain Res 2001;922:299-304.

[8] Cohen HB, Dement WC. Sleep: changes in threshold to electroconvulsive shock in rats after deprivation of "paradoxical" phase. Science 1965;150(3701): 1318-9.

[9] Cohen H, Thomas J, Dement WC. Sleep stages, REM deprivation and electroconvulsive threshold in the cat. Brain Res 1970;19(2):317-21.

[10] Shouse MN, Sterman MB. 'Kindling' a sleep-disorder: degree of sleep pathology predicts kindled seizure susceptibility in cats. Brain Res 1983;271(1):196-200.

[11] Rossini PM, Barker AT, Berardelli A, Caramia MD, Caruso G, et al. Non-invasive electrical and magnetic stimulation of the brain, spinal cord and roots: basic principles and procedures for routine application. Report of a IFCN committee. Electroenceph Clin Neurophysiol 1994;91:79-92.

[12] Currà A, Modugno N, Inghilleri M, Manfredi M, Hallett M, Berardelli A Transcranial magnetic stimulation techniques in clinical investigation. Neurology 2002;59:1851-9.

[13] Kujirai T, Caramia MD, Rothwell JC, Day BL, Thompson PD, Ferbert A, et al Corticocortical inhibition in human motor cortex. J Physiol 1993:471:501-19.

[14] Ziemann U. Intracortical inhibition and facilitation in the conventional paired TMS paradigm. Electroencephalogr Clin Neurophysiol Suppl 1999;51:127-36.

[15] Ziemann U, Lonnek S, Steinhoff BJ, Paulus W. The effect of lorazepam on the motor cortical excitability in man. Exp Brain Res 1996;109:127-35.

[16] Ziemann U, Lonnek S, Steinhoff BJ, Paulus W. Effect of antiepileptic drugs on motor cortex excitability in humans: a transcranial magnetic stimulation study. Ann Neurol 1996;40:367-78.

[17] Civardi C, Boccagni C, Vicentini R, Bolamperti L, Tarletti R, Varrasi C, et al. Cortical excitability and sleep deprivation: a transcranial magnetic stimulation study. J Neurol Neurosurg Psychiatry 2001;71:809-12.

[18] Scalise A, Desiato MT, Gigli GL, Romigi A, Tombini M, Marciani MG, et al Increasing cortical excitability: a possibile explanation for the proconvulsant role of sleep deprivation. Sleep 2006;29(12):1595-8.

[19] Manganotti P, Palermo A, Patuzzo S, Zanette G, Fiaschi A. Decrease in moto cortical excitability in human subjects after sleep deprivation. Neurosci Lett 2001;304(3):153-6.

[20] Manganotti P, Bongiovanni LG, Fuggetta G, Zanette G, Fiaschi A. Effects of sleep deprivation on cortical excitability in patients affected by juvenile myoclonic epilepsy: a combined transcranial magnetic stimulation and EEG study. J Neurol Neurosurg Psychiatry 2006;77(1):56-60.

[21] Badawy RAB, Curatolo JM, Newton M, Berkovich SM, MacDonell RAL. Sleep deprivation increases cortical excitability in epilepsy: syndrome specific effects. Neurology 2006;67:1008-22.

[22] Priori A Oliviero A Donati E Callea L, Bertolasi L Rothwell JC Human handedness and asymmetry of the motor cortical silent period. Exp Brain Res 1999;128(3):390-6.

[23] Endo T, Roth C, Landolt HP, Werth E, Aeschbach D, Achermann P, et al Selective REM sleep deprivation in humans: effects on sleep and sleep EEG. Am J Physiol 1998;274(4 Pt 2):R1186-94. 
[24] Rechschaffen A, Kales A, editors. A manual of standardized terminology, techiniques and scoring system for sleep stages of human subjects. Washinghton (DC): Public Health Service, US Government Printing Office; 1968.

[25] Smith MJ, Keel BA, Greenberg BD, Adams LF, Schmidt PJ, Rubinow DA, et al. Menstrual cycle effects on cortical excitability. Neurology 1999:53:2069-72.

[26] Palmieri MG, Iani C, Scalise A, Desiato MT, Loberti M, Telera S, et al. The effect of benzodiazepines and flumazenil on motor cortical excitability in the human brain. Brain Res 1999;815(2):192-9.

[27] Desiato MT, Caramia MD. Towards a neurophysiological marker of amyotrophic lateral sclerosis as revealed by changes in cortical excitability. Electroencephalogr Clin Neurophysiol 1997;105(1):1-7.

[28] Stetkarova I, Leis AA, Stokic DS, Delapasse JS, Tarkka IM. Characteristics of silent period after transcranial magnetic stimulation. Am J Phys Med Rehabil 1994;73(2):98-102.

[29] Caramia MD, Gigli GL, Iani C, Desiato MT, Diomedi M, Palmieri G, et al Distinguishing forms of generalised epilepsy using magnetic brain stimulation. Electroencephalogr Clin Neurophysiol 1996;98:14-9.

[30] Caramia MD, Cicinelli P, Paradiso C, Mariorenzi R, Zarola F, Bernardi G, et al 'Excitability' changes of muscular responses to magnetic brain stimulation in patients with central motor disorders. Electroeceph Clin Neurophysiol 1991;81:243-50.

[31] Di Lazzaro V, Restuccia D, Oliviero A, Proficue P, Ferrara L, Insola A, et al. Magnetic transcranial stimulation at intensities below active motor threshold activates intracortical inhibitory circuits. Exp Brain Res 1998;119(2):265-8.

[32] Cantello R, Granelli M, Civardi C, Mutani R. Magnetic brain stimulation: the silent period after the motor evoked potential. Neurology 1992:42(10):1951-9.

[33] Fuhr P, Agostino R, Hallet M. Spinal motor neuron excitability during the silent period after cortical stimulation. Electroenceph Clin Neurophysiol 1991;81: 257-62.

[34] Chen R, Lozano AM, Ashby PM. Mechanism of the silent period of transcranial magnetic stimulation. Evidence from epidural recordings. Exp Brain Res 1999;128(4):539-42.

[35] Siebner HR, Dressnandt J, Auer C, Conrad B. Continuous intrathecal baclofen infusions induced a marked increase of the transcranially evoked silent period in a patient with generalized dystonia. Muscle Nerve 1998;21:1209-12.

[36] Xi MC, Morales FR, Chase MH. The motor inhibitory system operating during active sleep is tonically suppressed by GABAergic mechanisms during other states. J Neurophysiol 2001;86(4):1908-15.
[37] Luppi PH, Clement O, Sapin E, Peyron C, Gervasoni D, Léger L, et al. Brainstem mechanisms of paradoxical (REM) sleep generation. Pflugers Arch - Eur J Physiol 2012;463(1):43-52.

[38] Torterolo P, Morales FH, Chase MH. GABA-ergic mechanisms in the pedunculopontine tegmental nucleus of the cat promote active (REM) sleep. Brain Res 2002;944:1-9.

[39] Pal D, Mallick BN. GABA in pedunculopontine tegmentum increases rapid eye movement sleep in freely moving rats: possible role of GABA-ergic inputs from substantia nigra pars reticulata. Neuroscience 2009;164(2):404-14.

[40] Longordo F, Kopp C, Lüthi A. Consequences of sleep deprivation on neurotransmitter receptor expression and function. Eur J Neurosci 2009;29(9): 1810-9.

[41] Mallick BN, Singh A. REM sleep loss increases brain excitability: role of noradrenaline and its mechanism of action. Sleep Med Rev 2011;15(3):165-78.

[42] Fountain MB, Kim JS, Lee SI. Sleep deprivation activates epileptiform discharges independent of the activating effects of sleep. J Clin Neurophysiol 1998;15(1):69-75

[43] Calvo JM, Alvarado R, Briones R, Paz C, Fernandez-Guardiola A. Amygdaloid kindling during rapid eye movement (REM) sleep in cats. Neurosci Lett 1982;29(3):255-9.

[44] Shouse MN. State disorders and state-dependent seizures in amygdala-kindled cats. Exp Neurol 1986;92(3):601-8.

[45] De Gennaro L, Marzano C, Veniero D, Moroni F, Fratello F, Curcio G, et al. Neurophysiological correlates of sleepiness: a combined TMS and EEG study. Neuroimage 2007;36(4):1277-87.

[46] Kreuzer P, Langguth B, Popp R, Raster R, Busch V, Frank E, et al. Reduced intracortical inhibition after sleep deprivation: a transcranial magnetic stimulation study. Neurosci Lett 2011;493(3):63-6.

[47] Del Felice A, Fiaschi A, Bongiovanni GL, Savazzi S, Manganotti P. The sleepdeprived brain in normals and patients with juvenile myoclonic epilepsy: a perturbational approach to measuring cortical reactivity. Epilepsy Res 2011;96(1-2):123-31.

[48] Huber R, Mäki H, Rosanova M, Casarotto S, Canali P, Casali AG et al. Human cortical excitability increases with time awake. Cereb Cortex 2012. http:// dx.doi.org/10.1093/cercor/bhs014. 